\title{
An informant questionnaire and a standard cognitive test improved screening for dementia
}

\author{
Mackinnon A, Mulligan R. Combining cognitive testing and informant report to increase accuracy in screening for dementia. Am J \\ Psychiatry 1998 Nov;155:1529-35.
}

\section{Question}

Can an informant report questionnaire combined with a standard cognitive test improve performance in screening for dementia in clinical settings?

\section{Design}

A blinded comparison of diagnostic tests for dementia.

\section{Setting}

A geriatric hospital and a memory clinic in Geneva, Switzerland.

\section{Patients}

106 patients who were 62 to 98 years of age (mean age $80 \mathrm{y}, 72 \%$ women). Exclusion criteria included no informant, serious medical condition, or severe sensorimotor deficit.

\section{Description of tests and diagnostic standard}

The Mini-Mental State Examination (MMSE) and the Informant Questionnaire on Cognitive Decline in the Elderly (IQCODE, 16 item short form) in French. The diagnostic standard was dementia diagnosed according to DSM-IV criteria.

\section{Main outcome measures}

Sensitivity and specificity, which were calculated by constructing receiver operating characteristic curves.

\section{Main results}

58 patients met the DSM-IV criteria for dementia. Logistic regression showed that the MMSE and IQCODE scores were statistically significant predictors of dementia $(\mathrm{p}<0.001)$ and that each test provided non-redundant information in the prediction of dementia. The table shows the sensitivity, specificity, and likelihood ratios for each test and combination of tests. An IQCODE cutoff point of 4.0 or an MMSE cutoff point of 23 led to greater sensitivity without reducing specificity when compared with the MMSE alone $(\mathrm{p}<0.01)$ (table). The 2 tests were also combined using weighted sums derived by logistic regression. Using this method to classify patients as having a greater probability of being demented than non-demented, sensitivity was higher than for the MMSE alone, and specificity was not reduced (table).

\section{Conclusion}

Screening for dementia was improved by using both the Informant Questionnaire on Cognitive Decline in the Elderly and the Mini-Mental State Examination.

Test characteristics for diagnosing dementia in older patients*

\begin{tabular}{|c|c|c|c|c|}
\hline Test or combination of tests & $\begin{array}{l}\text { Sensitivity \% } \\
(95 \% \text { CI })\end{array}$ & $\begin{array}{l}\text { Specificity \% } \\
(\text { CI })\end{array}$ & $+L R$ & $-L R$ \\
\hline MMSE score $\leqslant 23$ & 76 (63 to 86$)$ & 90 (77 to 97$)$ & 7.60 & 0.27 \\
\hline MMSE score $\leqslant 26$ & 95 (86 to 99$)$ & $60(45$ to 74$)$ & 2.38 & 0.08 \\
\hline IQCODE score $\geqslant 3.6$ & $90(79$ to 96$)$ & 65 (49 to 78$)$ & 2.57 & 0.15 \\
\hline MMSE score $\leqslant 23$ OR & & & & \\
\hline IQCODE score $\geqslant 4.0$ & $93(83$ to 98$)$ & $81(67$ to 91$)$ & 4.89 & 0.09 \\
\hline MMSE score $\leqslant 25$ and & & & & \\
\hline IQCODE score $\geqslant 3.62$ & $86(75$ to 94$)$ & 85 (72 to 94$)$ & 5.73 & 0.16 \\
\hline $\begin{array}{l}\text { Weighted sum for probability } \\
\text { of caseness } \geqslant 0.50\end{array}$ & $93(88$ to 100$)$ & $85(72$ to 94$)$ & 6.20 & 0.08 \\
\hline
\end{tabular}

*MMSE $=$ Mini-Mental State Examination; IQCODE = Informant Questionnaire on Cognitive Decline in the Elderly. LRs calculated from data in article and are defined in the glossary.

Source of funding: no external funding.

For correspondence: Dr A Mackinnon, Mental Health Research Institute, Locked Bag 11, Parkville, Victoria 3052, Australia. Fax +61393875061.

\section{Commentary}

Dementia is a progressive syndrome characterised by cognitive decline, behavioural and psychological disturbances, and deterioration of occupational or social functioning. The primary care practitioner, who usually sees the patient first, should be able to detect the dementia syndrome, preferably in its earliest stage which is the key to better management.

To make a diagnosis (besides functioning detriment and decline from a previous state), memory impairment with at least aphasia, agnosia, apraxia, or executive functioning is required. To screen and stage cognitive status, a reliable tool is needed. The MMSE is a scale widely used by physicians. It provides a reliable overall cognitive score but has limited sensitivity for detecting mild impairment. ${ }^{1}$ It has even less capacity to detect dementia of the non-Alzheimer's type because it does not test for executive functioning.

This well designed study shows that screening for dementia using the IQCODE as a compensatory tool with the MMSE reduces false negative cases because it provides non-redundant information for both cognitive and thinking impairments. In this way, some of the main criteria for dementia are better investigated (ie, executive problems and decline from a previously higher level of functioning).

Although not an objective of this study, the authors also show the effectiveness of using the IQCODE. Physicians regularly make informal use of a relative's knowledge about the patient, and this is probably the method by which many patients are routinely classified in health services. IQCODE takes systematic advantage of that knowledge. Although using both tests improved sensitivity, this study does not show if using the MMSE and IQCODE is better than the MMSE alone to screen demented patients with executive disturbances, as in frontal lobe or subcortical ones, or if the MMSE and IQCODE just detect more patients with Alzheimer's disease.

Overall, this method of screening is useful in clinical practice for reducing false negative diagnoses.

Fernando E Taragano, MD CEMIC University Buenos Aires, Argentina

1 Tombaugh TN, McIntyre NJ.J Am Geriatr Soc 1992;40:922-35. 